\title{
Eficacia de los programas educativos para reducir el sobrepeso y obesidad en niños
}

\author{
Efficacy of educational programs to reduce overweight and obesity in children
}

\section{Sr. Editor:}

El sobrepeso y la obesidad, actualmente, es considerando como una enfermedad crónica y muchos investigadores lo consideran como una epidemia de siglo XXI: "Se traduce por un aumento excesivo de peso y se diagnostica mediante el índice de masa corporal (IMC), que es un indicador simple con lo cual se relaciona el peso y la talla" (1).

En los últimos años, la obesidad en niños se ha asociado con la aparición, a edades más tempranas, de trastornos metabólicos como las dislipidemias, alteración del metabolismo de la glucosa, diabetes, hipertensión, hígado graso, apnea del sueño, estrés e incluso 1a depresión (2). En el 2010, la Asociación Americana de Diabetes informó que el $25 \%$ de adolescentes con obesidad presentaba hipertensión (vs $4 \%$ en adolescentes con peso adecuado); el 39\% bajos niveles de HDL (vs $18 \%$ en adolescentes con peso adecuado); y el $46 \%$, niveles elevados de triglicéridos (vs $17 \%$ en adolescentes con peso adecuado). De igual manera, se ha probado que adolescentes con oligomenorrea y obesidad están en mayor riesgo de desarrollar síndrome de ovario poliquístico (3).

En el Perú, el estado nutricional de la población ha tenido un cambio gradual, el sobrepeso y la obesidad en los niños, han aumentado en forma progresiva debido a los cambios en la dieta y estilos de vida. Además, existen determinantes sociales que podrían influenciar en el desarrollo del sobrepeso y la obesidad, como son la educación, el sexo, la pobreza, el lugar de residencia, entre otros (4). Al respecto un estudio demostró que la educación está asociada inversamente al sobrepeso y la obesidad (4).
Nosotros hemos contrastado la información sobre la efectividad de los programas para reducir el sobrepeso y obesidad en países hispanoamericanos incluyendo al Perú. En España, en un estudio en escolares se determinó como efectiva la intervención educativa a través de la reducción del índice de masa corporal (IMC) (5). Otro estudio español sobre programas de intervención educativos resultó en una disminución estadísticamente significativa del IMC de 1,14 a 1,02 al implementar los programas saludables (6). En Chile, el programa educativo con 2527 escolares redujo la obesidad en 21,8\% (7). En Perú, en el 2008 existía valores de $10 \%$ y $5,6 \%$ de sobrepeso y obesidad, respectivamente; una intervención a nivel local en un centro educativo basada en alimentación saludable y ejercicio físico por un periodo de 11 meses logró bajar el IMC de 64,8 \% a 58,5 \% siendo estadísticamente significativa esta reducción. En Lima, Perú, la efectividad en bajar la tasa de sobrepeso con los programas fue de $28,4 \%$ a $18,9 \%$ y la obesidad de $24,4 \%$ a $20,9 \%$, respectivamente (8).

El programa basado solo en actividad física 3 veces por semana, con 30 minutos cada sesión, durante el periodo escolar, ha dado buenos resultados en niños entre 9 y 11 años, pudiendo disminuir su IMC. Según estudios previos al parecer la actividad física da mejores resultados que solo la intervención a través de charlas de cortos tiempo (9).

Otros trabajos previos mencionaron que la efectividad de los programas en menores de edad entre 5 a 10 años, estuvo vinculado a la buena dieta y las actividades físicas, disminuyendo el IMC en un promedio de $47,3 \%$ en un total de 28 estudios (10).

\footnotetext{
Escuela de Nutrición, Universidad Privada del Norte. Lima, Perú

Facultad de Ciencias de la Salud, Universidad Privada del Norte. Lima, Perú

Estudiante de la carrera de nutrición

Maestro en Ciencias
} 
Por lo tanto, es probable que los programas educativos de promoción y prevención de la salud, si son bien orientados y estructurados, contribuyen a una mejora. Los programas que dan mejores resultados son los integrales donde se involucra a los centros educativos y a los padres de familia, por un periodo promedio de 12 meses, enfatizando en la actividad física además de ser sostenidos en el tiempo.

\section{Shirley Olivos Chuquino ${ }^{1, a}$, Jesus Eduardo Rojas Jaimes ${ }^{2, b}$}

\section{Correspondencia:}

Jesús Rojas Jaimes

Correo: jesus.rojas.jaimes@gmail.com

Dirección: Av. El Sol 461, San Juan de Lurigancho, Lima 15094, Perú

Teléfono: 511993638

\section{REFERENCIAS BIBLIOGRÁFICAS}

1. Herscovici C, Kovalskis I. Childhood obesity. A review of school-based preventive interventions. Rev Mex trastor aliment. 2015; 6(1):143-151.

2. Lobstein T, Baur LA, Jackson-Leach R. The Childhood Obesity Epidemic. In: Waters E, Swinburn BA, Seidell JC, and Uauy R. Eds. Preventing Childhood Obesity: Evidence Policy and Practice. Oxford: Wiley-Blackwell; 2010. p. 3-14.

3. American Diabetes Association. Standards of Medical Care in Diabetes-2010. Diabetes Care. 2010; 33(Suppl 1):S11-S61.

4. Alvarez Dongo D, Sanchez Abanto J, Gomez Guizado G, Tarqui Mamani C. Sobrepeso y obesidad: Prevalencia y determinantes sociales del exceso de peso en la población peruana. Rev Peru Med Exp Salud Pública; 2010; 29(3):303-313.

5. Aguilar Cordero M, Gonzáles Jiménez E, García García C, García López A, Álvarez Ferre J, Padilla López C, Ocete Hita E. Obesidad de una población de escolares de Granada: evaluación de la eficacia de una intervención educativa. Nut Hosp. 2011: 26(3);636-641.

6. Pérez Solís D, Álvarez Caro F, Suárez Tomás I, Suárez MenéndezE, Riaño Galán I, Díaz Martín J. Efectividad de una intervención escolar contra la obesidad. An de Ped. 2015; 83(1): 19-25.

7. Ratner GR, Durán AS, Garrido LM, Balmaceda HS, Jadue HL, Atalah SE. Impacto de una intervención en alimentación y actividad física sobre la prevalencia de obesidad en escolares. Nut Hosp. 2013; 28(5): 1508-1514.

8. Gago, J, Rosas O, Huayna, M, Jimenez D, Cordova F, Navarro A, Ramos W. Efectividad de una intervención multisectorial en educación alimentarianutricional para prevenir y controlar el sobrepeso y la obesidad en escolares de dos Instituciones Educativas Públicas del Distrito de Villa el Salvador. Rev peru Epidemiol. 2014; 18(3):1-7.

9. Pumar Vidal B, Navarro Paton R, Basante Camiño S. Efectos de un programa de actividad física en escolares. Ed Fís y Cien. 2015; 17(2):1-13

10. Visiedo A, Sainz de Baranda P, Crone D, Aznar S, Perez Llamas F, Sanchez Jimenez R, Zamora S. Programas para la prevención de la obesidad en escolares de 5 a 10 años. Revisión Sistemática. Nut Hosp 2016; 33(4):814-824.

Recibido: 28/12/2019

Aceptado: 30/01/2020 